\title{
La fragmentation des systèmes scolaires nationaux à l'heure de la mondialisation
}

Introduction

Introduction. The fragmentation of national school systems in the era of globalization

Introducción. La fragmentación de los sistemas escolares nacionales a la hora de la mundialización

Anne Barrère et Bernard Delvaux

\section{OpenEdition} Journals

Édition électronique

URL : https://journals.openedition.org/ries/6038

DOI : $10.4000 /$ ries.6038

ISSN : 2261-4265

Éditeur

France Education international

Édition imprimée

Date de publication : 1 décembre 2017

Pagination : $39-50$

ISBN : 978-2-85420-616-6

ISSN : $1254-4590$

Référence électronique

Anne Barrère et Bernard Delvaux, « La fragmentation des systèmes scolaires nationaux à l'heure de la mondialisation », Revue internationale d'éducation de Sèvres [En ligne], 76 | décembre 2017, mis en ligne le 01 décembre 2017, consulté le 25 juin 2021. URL : http://journals.openedition.org/ries/6038 ; DOI : https://doi.org/10.4000/ries.6038 


\title{
La fragmentation des systèmes scolaires nationaux
}

\section{Introduction}

\section{La fragmentation des systèmes scolaires nationaux à l'heure de la mondialisation}

\author{
Anne Barrère \\ Université Paris-Descartes \\ Bernard Delvaux \\ Université catholique de Louvain
}

La modernité a correspondu à la montée en puissance des États-nations et à l'emprise croissante de ceux-ci sur l'éducation des enfants et des jeunes. Au fil de leurs histoires singulières, les États ont développé cette emprise en établissant un contrôle sur des organisations scolaires privées et en multipliant les écoles publiques. Ils ont ainsi enrôlé dans ce mode d'éducation particulier un nombre croissant d'enfants et de jeunes pendant un nombre croissant d'années de vie. Ce processus d'expansion et d'unification des organisations scolaires est inégalement abouti, mais dans la plupart des États, les écoles sont devenues des éléments centraux de l'éducation des enfants et des jeunes, au point qu'il est devenu commun - bien qu'abusif - de parler de systèmes éducatifs pour désigner des systèmes scolaires qui ne constituent pourtant qu'un sous-ensemble du champ éducatif.

L'hypothèse sous-tendant ce dossier est que des processus de fragmentation déstructurent les systèmes scolaires nationaux les plus solidement établis, tandis qu'ils freinent les efforts d'unification des États disposant de systèmes scolaire peu unifiés. On peut en outre faire l'hypothèse que la prééminence des systèmes scolaires dans les champs éducatifs tend à s'éroder lentement, sous les coups de butoir d'initiatives multiples de familles, d'associations, de communautés, d'entreprises ou de fondations privées. On voit se multiplier les offres de formation, stage, coaching, soutien scolaire, scolarité à domicile, cours à distance et autre shadow education. Ces initiatives externes peuvent être vues comme des vecteurs de fragmentation des systèmes scolaires proprement dits. Ce dossier n'en traitera cependant pas. Nous avons en effet choisi de nous centrer sur les systèmes scolaires au sens strict, et plus précisément sur ce qui est souvent qualifié d'enseignement obligatoire (primaire et secondaire). Les organisations 
constitutives de ces systèmes scolaires partagent les attributs essentiels de ce qu'est une école, à savoir un lieu clos, réservé à des collectifs d'enfants et de jeunes, exclusivement dédié à l'apprentissage et à l'éducation, caractérisé en outre par une planification centralisée des apprentissages mis en œuvre par des adultes spécifiquement formés à ce travail. Mais le partage de tels attributs ne suffit pas à constituer un système scolaire unifié. L'unification suppose aussi le partage de finalités, de contenus et de modèles éducatifs. C'est précisément sur ces dimensions que les systèmes scolaires nationaux se fragmentent progressivement ou cessent de s'unifier.

\section{DES PROCESSUS DE MONDIALISATION VECTEURS DE FRAGMENTATION DES SYSTÈMES SCOLAIRES NATIONAUX}

Ce processus de fragmentation des systèmes scolaires nationaux, couplé à l'affaiblissement de leur prééminence dans le champ éducatif, peut sembler paradoxal à l'heure où d'évidents processus de globalisation et de mondialisation affectent les sociétés et leur champ éducatif. À l'heure aussi où de nombreuses études montrent, de manière convaincante, la force unificatrice d'organisations internationales telles que l'OCDE (Ozga, 2012). Comment, dès lors, penser ensemble ces processus de mondialisation et de fragmentation ? Comment articuler une hypothèse de propagation mondiale de modèles, dispositifs, phénomènes ou discours auparavant limités à une région à une hypothèse de déstructuration de systèmes unifiés? L'idée que nous défendons est que les concepts de forme scolaire (Vincent, 1980) et de système scolaire national, qui se sont mondialisés tout au long de la modernité, sont désormais touchés par des processus de mondialisation qui participent à leur remise en cause.

Indéniablement, la forme scolaire s'est mondialisée tout au long de la modernité, d'abord sous l'impulsion d'acteurs transnationaux (les congrégations religieuses, notamment) ensuite relayés ou accompagnés par les États occidentaux. Ceux-ci ont travaillé à l'unification de leurs propres systèmes éducatifs nationaux. Ils ont également exporté dans le monde la forme scolaire mais aussi l'idée de systèmes scolaires unifiés à l'échelle nationale. Ce sont ces systèmes scolaires nationaux qui se fragmentent désormais en interne (ou cessent de s'unifier) et voient certains de ces fragments s'émanciper de la forme scolaire.

Ce processus de fragmentation n'est pas incompatible avec l'existence de processus de mondialisation. Nous défendons même l'idée qu'elle en est en partie le produit. Certains de ces processus conduisent les acteurs partageant un même territoire à ne plus vouloir ou pouvoir élaborer à l'échelle nationale des compromis sur les questions éducatives (Delvaux, 2015). D'autres contribuent à l'émergence d'acteurs supra ou multinationaux qui viennent concurrencer les 
instances étatiques ou se substituer à elles. Alors que les premiers processus affaiblissent durablement la capacité des États à maintenir ou accroître l'unité de leur système scolaire, les seconds structurent en partie la recomposition de ces fragments autour de réseaux multinationaux d'écoles.

Qu'en est-il de la première série de processus? Même si on observe actuellement des phénomènes de repli communautaire sur des territoires nationaux ou infranationaux, ces territoires abritent des populations plus hétérogènes qu'auparavant, tant au niveau de leurs ressources (les inégalités intra-nationales s'accroissent) qu'au niveau de leurs références culturelles ou religieuses, ou de leurs projets et attentes. Se mondialisent donc simultanément une diversification des populations et ce qu'elle suscite chez certains, à savoir un mouvement de repli communautaire, et ces deux phénomènes sont vecteurs d'une demande pour des écoles différenciées. Cette demande a également deux autres ressorts ; d'une part, l'aspiration à l'autonomie individuelle et le refus d'une assignation à une "identité pour la vie», qui se diffusent dans toutes les sociétés et fondent les revendications parentales de libre choix d'école et de demandes juvéniles d'individualisation des trajectoires de vie; d'autre part, la perception que, dans des sociétés hétérogènes et interdépendantes, les pouvoirs politiques nationaux sont affaiblis, et qu'il est donc plus efficace d'investir dans des stratégies de choix individuels ou de création/transformation d'offres éducatives locales que dans des actions collectives visant à généraliser à l'échelle nationale un modèle éducatif particulier. Ainsi, ces quatre processus mondialisés que sont l'hétérogénéisation des populations, le repli communautaire, l'aspiration à l'autonomie individuelle et le désenchantement par rapport au pouvoir politique national compromettent les efforts d'unification des systèmes scolaires nationaux et favorisent l'émergence de nouvelles offres éducatives extra-scolaires.

Cette fragmentation interne des systèmes scolaires nationaux est renforcée par la multiplication d'acteurs éducatifs mondialisés. On voit en effet se développer des réseaux, des associations, des entreprises et des " philentrepreneurs » éducatifs (Olmedo et Ball, 2011) agissant à des échelles multinationales alors que, dans le même temps, des organisations associant des États (Unesco, OCDE, Union européenne, Isesco, etc.) œuvrent au rapprochement des systèmes nationaux, sans pour autant toujours soutenir l'unification interne de ces systèmes. Plusieurs d'entre elles relaient en effet les rhétoriques néo-libérales qui promeuvent la marchandisation de l'éducation et une plus large autonomie des écoles, ce qui est de nature à encourager plus qu'à freiner les initiatives des réseaux d'organisations qui créent, soutiennent ou affilient des établissements scolaires se distinguant des écoles «types» du pays. Cette idéologie est à ce point prégnante qu'elle pousse des États pourtant bien classés dans les évaluations internationales des systèmes scolaires à ouvrir des espaces pour des initiatives privées, comme le montre dans ce dossier Signe Bock Segaard, à propos de la Suède, où les établissements scolaires à but lucratif ont été autorisés et scolarisent aujourd'hui près d'un élève sur dix. 
Désormais, les réseaux internationaux d'écoles ne sont plus seulement le fait de congrégations religieuses (ou d'États promouvant leur curriculum national dans les pays où vivent leurs ressortissants). Des réseaux fédèrent des acteurs détenant peu de capitaux économiques tandis que d'autres se constituent à l'initiative de macro acteurs économiques qui poursuivent des buts de profit ou agissent via des fondations. Ces réseaux rassemblent des écoles s'affichant comme étant "traditionnelles» ou "alternatives», des écoles ciblant une communauté particulière (religieuse ou ethnique), des écoles préparant à un même diplôme international ou encore des écoles destinées aux publics les plus pauvres, ce dernier type de réseaux pouvant être investi par des ONG ou des "philentrepreneurs" mais aussi par des entreprises privées à but lucratif qui développent des low cost schools (Caerus capital, 2017) ${ }^{1}$. Certains de ces réseaux comptent plus de 10000 écoles, soit bien plus que les anciens réseaux des congrégations catholiques ${ }^{2}$. Mais aucun n'est, à l'heure actuelle, en passe de s'imposer mondialement : même les plus importants d'entre eux ne regroupent qu'une proportion marginale des écoles existant dans le monde ${ }^{3}$. En outre, ils sont le plus souvent inégalement répartis au plan géographique. Mais ces réseaux sont des acteurs importants des processus en cours. Encore trop peu étudiés et donc relativement méconnus, ces réseaux paraissent moins homogènes que les systèmes scolaires nationaux ou que les congrégations religieuses internationales de jadis: ils exercent un moindre contrôle sur leurs membres, ce qui génère souvent une différenciation interne non négligeable, comme le montre bien l'article de Marie-Laure Viaud à propos des écoles Montessori. Mais cette souplesse constitue aussi leur force d'attraction à une époque où les acteurs hésitent à s'encarter durablement dans des organisations structurées et hiérarchisées. Moins « contrôlants ", ces réseaux favorisent l'émergence d'entrepreneurs au sein des divers systèmes scolaires, en leur donnant des appuis logistiques et rhétoriques et en en faisant des ferments d'une fragmentation qui s'opère sur deux axes, l'un lié à la stratification sociale et l'autre à la différenciation des modèles et projets éducatifs.

\section{UNE FRAGMENTATION LIÉE À LA STRATIFICATION SOCIALE}

Un premier niveau de fragmentation transversal peut se lire au travers de la manière dont les trajectoires de scolarisation s'organisent et se différencient sur un territoire donné mais aussi au travers de la préoccupation forte des élèves

\footnotetext{
1. Par exemple, les low cost schools développées par les entreprises privées Bridge international academies ou Omega schools, ou encore la chaîne des Innova schools au Pérou.

2. On compte par exemple environ 850 écoles jésuites dans le monde.

3. Parmi les réseaux les plus développés, citons Montessori (20000 écoles), Cambridge Assessment International Education (10000), les écoles associées de l'Unesco (10000) ou Baccalauréat international (4500). Même si une évaluation chiffrée est difficile à effectuer, il est certain que le plus gros de ces réseaux ne représente pas plus de $0,5 \%$ des écoles existant dans le monde.
} 
et des familles quant à la qualité de l'offre scolaire proposée, souvent jaugée en fonction de son efficacité en matière d'accès à un niveau d'éducation ou à l'emploi.

Dans de nombreux pays, l'accès à la scolarisation primaire et secondaire reste l'objet de grandes inégalités, voire d'une dualisation du système entre écoles publiques gratuites et écoles privées payantes. Dans d'autres, où un système scolaire unifié entendait précisément contenir ou réduire ces inégalités, la permanence voire le creusement des inégalités fait douter de l'action publique, aboutissant à des critiques et remises en question, mais aussi à des appels à d'autres types d'action, plus efficaces, ciblant les publics et territoires particulièrement touchés par ces inégalités. Ce sont ainsi indissociablement l'évaluation des écarts entre les conditions de scolarisation de divers publics scolaires et la force d'adhésion au caractère nuisible, pour les individus et les sociétés, de trop brutales inégalités, qui nourrissent une vision en termes de fragmentation et les appels à l'inclusion.

Ainsi trouve-t-on, à l'intérieur des systèmes publics eux-mêmes, une part des facteurs favorisant la fragmentation. C'est ce que montre l'article de Michael Merry sur les écoles religieuses en Europe, dont l'avenir assuré procède, selon lui, du traitement souvent peu favorable des scolarisations dans les établissements concentrant une majorité de publics issus des minorités, traitement qui constituerait, au-delà de critères de conviction ou de commodité, un des éléments conduisant une part de ces populations à choisir des écoles privées confessionnelles, parce qu'elles considèrent ne pouvoir accéder qu'à une offre publique de rabais.

Mais ce sont aussi parfois des politiques publiques officiellement conçues pour réduire ce type de fragmentation qui peuvent être soupçonnées de le nourrir. Daniel Frandji analyse ainsi les ambivalences de deux politiques françaises récentes : d'une part l'inflexion des politiques d'éducation prioritaire dans le sens de la prise en compte de catégories particulières d'élèves, plutôt unificatrice; d'autre part la réforme des rythmes scolaires dans l'école primaire, se traduisant par le recours accru à des activités éducatives péri-scolaires, plutôt génératrice de fragmentation. Ainsi, la circulation entre les situations des différents pays atteste d'un débat, plus ou moins formalisé, plus ou moins vif, sur les politiques à mettre en ouvre face aux inégalités. Faut-il encourager la diversification de l'offre éducative pour prendre en charge les publics les plus fragilisés ou au contraire faut-il craindre que cette prise en charge n'accentue encore les différences, conduisant à une dualisation selon les publics accueillis, nourrissant la fragmentation liée à la stratification sociale?

L'article portant sur les États-Unis est emblématique de ce débat. À la Nouvelle-Orléans, Frank Adamson montre que l'expérience des charter schools, née officiellement du désir de stimuler l'innovation dans le système public et de bénéficier aussi aux publics les plus faibles, manifeste son inefficacité à réduire les inégalités. La comparaison avec le Massachusets sert par ailleurs à prendre la 
mesure des variations considérables entre États américains sur ce thème. La fragmentation peut être plus ou moins officialisée, plus ou moins institutionnalisée sous des statuts différents, plus ou moins autorisée et régulée par la juridiction compétente. Mais désormais, l'idée d'une hiérarchisation complexe d'offres éducatives inégales, dépendant fortement des caractéristiques des publics d'élèves, fait converger des systèmes scolaires nationaux qui n'ont jamais pu unifier d'offre publique organisée et majoritaire et des systèmes qui l'ont historiquement connue mais voient cette offre publique faire face à la pression sociale exercée sur la formation initiale et la diplomation.

Si ces constats rejoignent des principes de différenciation très anciens, et au premier chef l'opposition privé/public, le processus aboutit à prendre en compte de manière croissante les variations intra secteurs. Ni le secteur public ni le secteur privé aujourd'hui ni même - comme le montre Marie-Laure Viaud les réseaux unifiés par un projet alternatif apparemment homogène comme celui du réseau Montessori n'échappent à des enjeux de différenciation venus des choix scolaires et des inégales ressources des acteurs face à ces choix.

\section{UNE FRAGMENTATION DES MODÈLES ET PROJETS ÉDUCATIFS}

Le second axe de fragmentation se réfère aux finalités, aux formes éducatives, ou simultanément à ces deux dimensions, ce que dans un langage durkheimien on appellerait "projet éducatif », en ce qu'il suppose la réflexion sur le type d'individu formé par l'école, et l'articulation plus ou moins précise de ces réflexions avec des modalités d'enseignement. Comment s'opère ici la différenciation, et pourquoi la qualifier de fragmentation? Un premier plan concerne l'échelle de référence du projet éducatif, qui désormais ne fait plus seulement référence à l'échelon national mais aussi à des échelons locaux ou supranationaux, qu'il s'agisse de communautés religieuses, pédagogiques, ou de projet d'un curriculum mondialisé (Morin, 2007), particulièrement explicite dans le Baccalauréat international, dont Tristan Bunnell retrace l'histoire dans ce dossier. Un certain nombre d'offreurs éducatifs entendent ainsi dépasser des cultures scolaires essentiellement définies comme nationales, pour proposer une adaptation (ou une résistance) au processus de globalisation économique et culturelle.

Un autre plan de différenciation se réfère à des différences culturelles de groupes minoritaires, appuyées ou non sur des valeurs explicitement revendiquées. Ce qui peut être perçu comme un véritable déficit éducatif par certaines familles donne lieu à des choix d'adhésion à une certaine vision de l'enfant et de ses droits, tout comme à la nécessité de former la créativité ou de prendre en compte l'importance des familles dans la vie de l'école. Il peut aussi s'agir de l'adhésion à un projet d'action sur le monde, par exemple dans le cadre de l'affirmation de valeurs écologiques ou démocratiques (comme dans le cas du réseau 
des écoles Sudbury, qui ne fait pas l'objet d'un article dans le présent numéro, mais dont le succès actuel est grandissant en France). Il peut enfin s'agir d'un projet centré sur la restauration de valeurs traditionnelles, jugées bafouées ou trop oubliées.

Dans bien des cas, ces postures sont aussi très concernées par la mise en œuvre concrète de ces valeurs dans des dispositifs pédagogiques particuliers (organisation de la journée scolaire, matériel utilisé, types de règlements, etc.). En ne s'en tenant pas à des déclarations d'intention, ces acteurs interrogent la forme scolaire. La différenciation de l'offre, sur cet axe, est d'autant plus explicite et assumée que les porteurs de ces propositions sont souvent des militants animés également par une critique culturelle de ce qui est alors perçu comme une trop grande standardisation ne correspondant pas à des sociétés marquées par la nécessité de fabriquer des individus singuliers (Martuccelli, 2011). L'article de Jean-Emile Charlier, Sarah Croché et Oana Marina Panait, analysant la situation éducative au Sénégal, montre d'ailleurs comment cette fragmentation n'épargne nullement les offres religieuses elles-mêmes. On y repère en effet l'existence de deux modèles d'écoles coraniques, différentes dans leurs vision et traitement de l'enfant, venant fissurer l'idée d'une offre unifiée du projet éducatif de formation de l'individu musulman.

Ce processus de fragmentation des modèles et projets éducatifs n'est pas sans rapport avec la difficulté d'affirmation d'un projet éducatif fort dans bien des systèmes publics d'éducation, et dans l'absence d'incarnation curriculaire et pédagogique forte du projet d'égalité des chances, parfois davantage associé à des changements morphologiques structurels qu'à des propositions faisant retour sur les programmes ou la relation pédagogique (Barrère, 2011). Dans ce contexte, l'avenir de cette fragmentation se joue également dans la manière dont le projet d'offre « égalitariste et universaliste » de l'école publique coexiste avec d'autres propositions, comme dans le cas du Sénégal, où l'idée - portée en particulier par l'Unesco et la Banque mondiale - prévaut que tous les opérateurs éducatifs peuvent et doivent être considérés, y compris ceux porteurs de types d'éducation les plus informels.

\section{ARTiculation des deuX Types DE FRAGMENTATION ET DE LEURS CRITIQUES RESPECTIVES}

Il est évidemment possible de considérer le second processus dans la seule dépendance du premier, tant la différenciation de l'offre sur le plan des finalités et modalités éducatives paraît rejoindre parfois des préoccupations stratégiques, servant les intérêts de groupes sociaux cherchant des formes d'entresoi et l'accès aux positions hautes de la stratification sociale. Ainsi, le Baccalauréat international attire-t-il surtout les publics visant des trajectoires 
internationales et se mondialise-t-il plus largement partout ailleurs qu'en Afrique, les réseaux Montessori séduisent-ils surtout les classes moyennes urbanisées, les écoles privées sous contrat servent-elles encore et toujours à contourner des établissements publics trop peu performants et fréquentés par les milieux populaires.

Pourtant cette vision est réductrice, précisément parce que, désormais, ces offres éducatives actuellement minoritaires affrontent aussi la question des inégalités et de la ségrégation potentielle des publics, et parce que les projets affirmés sont susceptibles d'interprétations complexes, comme le montre l'étonnant appui du gouvernement équatorien de gauche au Baccalauréat international. Les articles concernant deux pays aussi différents que la Corée du Sud et le Pérou en attestent. La situation péruvienne décrite par Martin Santos est celle d'inégalités jamais résorbées, incarnées largement par une dualité public-privé, mais également travaillées par une fragmentation culturelle à l'intérieur des deux secteurs : alors que l'offre publique comporte aussi bien des collèges d'excellence que des écoles rurales bilingues à destination des communautés indiennes, l'offre privée croissante, représentant quasiment la moitié des écoles à Lima, mélange des écoles "low cost» dans les quartiers les plus pauvres et des collèges d'élite. Il existe aussi des établissements confessionnels résultant d'une association privé-public comme ceux du réseau jésuite "Alegria y Fe ». Dans le cas de la Corée du Sud, décrit par Chae-chun Gim, la critique faite à une offre publique homogène conduit à des appels périodiques à l'individualisation de parcours plus respectueux des talents et des besoins de chacun, dans des écoles secondaires plus spécialisées, centrées sur les langues, les arts ou les sciences, individualisation que le débat public, dans un balancier bien décrit par l'auteur, soupçonne de nourrir inégalités et surenchère de compétition, ouvrant ainsi la voie à des politiques plus unificatrices.

L'opposition entre secteur public et privé peut ainsi soit se durcir, au travers de situations de compétitions accrues sur des marchés scolaires, soit se brouiller, lorsque l'on prend en considération l'existence d'un privé de « réparation " ou destiné aux publics les plus fragiles ou en échec. Surtout, les offres étatiques d'éducation publique, même si elles peuvent toujours à juste titre dénoncer les risques d'éclatement ou les déficits de cohésion, parfois exprimés par le soupçon de "communautarisme ", à l'égard de ceux qui défendent des modèles et projets éducatifs alternatifs, peinent parfois à légitimer le lien entre justice scolaire optimale et offre indifférenciée. Mais si elles peinent à convaincre, c'est aussi parce que leur manque d'affirmation sur le plan éducatif peut donner parfois l'impression qu'elles laissent, en fait, se développer une version éducative du « TINA ${ }^{4}$ ». Il n'y aurait ainsi pas d'alternative à une situation où l'élève et sa famille seraient responsables de leurs choix éducatifs, les enseignants et les établissements de leurs résultats et où la méritocratie scolaire serait digérée par

4. There is no alternative. 
la logique de la compétition généralisée, où il est normal que le meilleur gagne (Dubet, 2004). Ainsi peut-on avoir l'impression de faire face à un débat certes diffus et informel, mais lui aussi largement mondialisé, sur les finalités de l'éducation, où certains défendent des modèles éducatifs plus éloignés des demandes économiques, pour faire de l'école, au moins pendant un certain temps du curriculum obligatoire, un espace débarrassé des considérations de compétition.

Les articles du dossier tendent également à remettre en question le contraste entre des pays où un projet d'éducation nationale aurait vocation à réduire la fragmentation - modèle largement exporté et diffusé par les pays «du Nord»- et des pays qui auraient renoncé à le faire. Les situations sont désormais largement plus incertaines, et du coup partagées, généralisant les questions autour du rôle joué par l'école dans la construction de la cohésion sociale et culturelle des sociétés nationales.

\section{VERS UNE FRAGMENTATION DURABLE ?}

Si ce dossier consolide l'hypothèse de processus de fragmentation affectant tous les systèmes scolaires nationaux, il ne permet pas de prédire ce sur quoi va déboucher ce processus. Nous pensons toutefois qu'il est possible d'esquisser deux scénarios idéal-typiques.

Le premier scénario verrait s'établir durablement une situation de fragmentation des systèmes scolaires nationaux. Chacun de ceux-ci céderait la place à un champ éducatif morcelé, où se côtoieraient des offres adoptant plus ou moins la forme scolaire actuelle et d'autres rompant avec celle-ci, des offres également diversifiées quant aux finalités éducatives qu’elles poursuivraient et aux publics qu'elles recruteraient. Un champ où cohabiteraient des acteurs privés, avec ou sans but lucratif, et un secteur public qui privilégierait cependant en son sein l'autonomisation des projets éducatifs locaux, autorisant les établissements à se singulariser aussi bien sur l'axe de la stratification sociale que sur l'axe des modèles et projets éducatifs. Dans ce scénario, les jeunes et leurs parents seraient responsables des choix qu'ils effectueraient sur ce "marché " diversifié d'offres éducatives. Ce serait à eux de construire pas à pas un parcours d'apprentissage et de vie, d'enchaîner ces modules d'apprentissage indépendants en fonction de l'histoire singulière de l'apprenant et des éventuels prérequis (ou paiements) exigés pour l'accès à certains modules. Dans un tel scénario, où chacun des systèmes nationaux dériverait vers une fragmentation accrue, l'offre éducative tendrait à se mondialiser, avec de puissants offreurs multinationaux et des apprenants libres d'aller chercher des offres en dehors du périmètre national. On voit mal comment un tel système ne renforcerait pas les inégalités. Mais sa force réside dans le fait qu'il ne nécessiterait plus un consensus normatif au sein d'un territoire : dans un tel scénario, l'ordre social ne reposerait «plus tant sur 
des valeurs partagées mais bien davantage sur divers conventions ou mécanismes de coordination qui, pour fonctionner, ne nécessitent pas de consensus normatif : l'argent, les diplômes, les contrats, les référentiels, les standards sont autant de moyens qui, au sein d'un domaine particulier, permettent à des personnes de se coordonner entre elles sans véritable consensus normatif " (Mangez et al., 2017).

Bien que ce scénario paraisse aujourd'hui le plus probable parce qu'il s'inscrit dans la continuité des tendances que ce dossier met à jour, un second scénario mérite néanmoins d'être évoqué. On y verrait se reconstituer de nouveaux systèmes éducatifs relativement homogènes, à des échelles territoriales ne correspondant pas nécessairement aux États mais à des entités se constituant à des échelles territoriales plus petites ou plus larges que ces États. Cela supposerait que se dégage au sein de ces entités territoriales un consensus sur un projet éducatif substantiel et pas seulement sur quelques principes minimalistes de régulation d'acteurs laissés largement autonomes, autrement dit qu'aboutisse une définition partagée du type d'être humain et de société auquel devrait contribuer le système scolaire (ou, plus largement, un système éducatif dont l'école ne serait qu'une composante). Cet énoncé montre à lui seul qu'un tel scénario suppose toute une série de conditions dont certaines paraissent peu probables et dont la conjonction paraît encore bien moins plausible. On mesure donc le défi que constitue la recherche active d'un tel scénario, à l'heure où les institutions politiques sont faibles, où les dispositifs de décision démocratique sont à réinventer et où les liens d'interdépendance entre territoires sont étroitement tissés. Mais il n'est pas interdit d'imaginer que les tensions sociales engendrées par le premier scénario dépassent certaines limites et qu'un des modèles éducatifs alternatifs actuellement en compétition soit adopté dans un territoire circonscrit qui aurait le pouvoir d'en contaminer d'autres... Encore faudrait-il qu'un tel modèle éducatif alternatif puisse parler à de larges franges des populations partageant un même territoire, ce qui implique selon nous qu'il parvienne à incarner dans des dispositifs éducatifs concrets un nouvel équilibre entre l'idéal d'émancipation et l'exigence de solidarité, ainsi qu'entre l'éducation à l'expression de l'individualité et l'éducation à participer au travail collectif d'orientation d'une histoire commune (Tout Autre Chose, 2016). Pour qu'un tel projet émerge, il est impératif qu'un nombre croissant de chercheurs et d'intellectuels s'intéressant aux questions d'éducation cessent de circonscrire ces questions au seul système scolaire et qu'ils s'attachent à comprendre sous un nouvel angle de vue ce qui est souvent abordé par l'unique prisme des marchés scolaires. C'est ainsi que pourrait se réinventer une question éducative portant sur les finalités et les modalités, sur le rapport à l'enfance et à la jeunesse ainsi que sur l'articulation entre école et société. 


\section{BIBLIOGRAPHIE}

BARRÈRE A. (2011) : L'éducation buissonnière, Paris, Armand Colin.

CAERUS CAPITAL (2017): The business of education in Africa. [https://goo.gl/ v1Z6oX]

DELVAUX B. (2014) : Une tout autre École, Pensées libres.

DUBET F. (2004) : L'école des chances, Paris, Seuil.

MANGEZ E., BOUHON M., CATTONAR B., et al. (2017) : "Faire société dans le monde de demain. Quel rôle pour l'école ? ", Les Cahiers de recherche du Girsef, 110.

MARTUCCELLI D. (2011) : La société singulariste, Paris, Armand Colin.

MORIN E. (2000) : Les sept savoirs du futur, Paris, Seuil.

OLMEDO A., BALL S. (2011) : "Nouvelle" philanthropie, capitalisme social et développement international. Les nouvelles façons de voir le don", Revue internationale d'éducation de Sèvres, 58, p. 119-131.

OZGA J. (2012) : «Assessing PISA », European Educational Research Journal, 11, 2, p. $166-171$.

TOUT AUTRE CHOSE, Manifeste pour une tout autre école. [https://goo.gl/68PkFu], consulté le 26 novembre 2017.

VINCENT G. (1980) : L'école primaire française. Étude sociologique, Presses universitaires de Lyon, Éditions des Sciences de l'Homme. 
